\title{
Herdabilidades e correlações entre pesos do nascimento à idade adulta em rebanhos da raça Nelore ${ }^{1}$
}

\section{Arione Augusti Boligon², Lucia Galvão de Albuquerque ${ }^{3}$, Maria Eugênia Zerlotti Mercadante ${ }^{4}$, Raysildo Barbosa Lôbo ${ }^{5}$}

\author{
1 Projeto de pesquisa financiado pela FAPESP. \\ 2 Pós-graduação em Genética e Melhoramento Animal, FCAV/UNESP - Jaboticabal, SP. Bolsista FAPESP. \\ ${ }^{3}$ Departamento de Zootecnia, FCAVIUNESP - Jaboticabal, SP. Pesquisador do CNPq e INCT-CA. \\ ${ }^{4}$ Estação Experimental de Zootecnia de Sertãozinho - IZ/APTA/SAA-SP, Caixa Postal 63, CEP: 14160-000, Sertãozinho, SP. \\ ${ }^{5}$ ANCP - Ribeirão Preto, SP.
}

RESUMO - Dados de 19.458 animais da raça Nelore, nascidos entre 1975 e 2002 e pertencentes a oito fazendas participantes do Programa de Melhoramento Genético da Raça Nelore (PMGRN), foram utilizados para estimar componentes de covariância, herdabilidade e correlações genéticas dos pesos ao nascimento, à desmama, ao sobreano e aos 2 , 3 e 5 anos de idade. Utilizou-se o método da máxima verossimilhança restrita, em análises uni, bi e multicaracterísticas, com modelos que incluíram o efeito genético aditivo direto, como aleatório, além dos efeitos fixos de grupo de contemporâneos e os efeitos linear e quadrático da idade do animal à pesagem (com exceção do modelo para peso ao nascer) e da idade da mãe ao parto. O efeito aleatório de ambiente permanente materno também foi incluído nos modelos de análise do peso ao nascer, peso à desmama e peso ao sobreano e o efeito aleatório genético materno somente no modelo do peso à desmama. As herdabilidades estimadas em análise multicaracterística para os pesos ao nascer, à desmama, ao sobreano, aos 2, 3 e aos 5 anos de idade foram 0,$25 ; 0,33 ; 0,34 ; 0,32 ; 0,33$ e 0,35 , respectivamente. As correlações genéticas estimadas entre os pesos foram positivas e de moderadas a altas magnitudes e tenderam a diminuir com o aumento da distância entre as pesagens, o que indica que a seleção para peso em qualquer idade deverá promover mudança genética nos pesos nas demais idades, inclusive no peso adulto de fêmeas da raça Nelore. As estimativas de herdabilidade obtidas na análise multicaracterística foram superiores, sobretudo para os pesos aos 3 e aos 5 anos de idade, o que sugere melhor partição das variâncias genética e de ambiente em comparação às análises uni e bicaracterísticas, porém a alta demanda de recursos computacionais em análises de muitas características pode ainda dificultar o uso dessas análises em grandes bancos de dados.

Palavras-chave: bovino de corte, correlação genética, peso adulto, peso corporal

\section{Heritability and correlations between weights from birth to maturity in Nellore cattle}

ABSTRACT - Records of 19,458 Nellore breed animals, born from 1975 to 2002, from 8 herds participating in the Nellore Cattle Breeding Program (NCBP) were used to estimate (co)variance components, heritability and genetic correlations for birth weight, weaning weight, weight after yearling and at 2, 3 and 5 years of age. The restricted maximum likelihood method, with univariate, bivariate and multivariate analyses, was used. The models included the random additive genetic direct effect (animal) and fixed effects of age group and the linear and quadratic effects of calf age (except for the birth weight model) and age dam at calving. The random effect of permanent maternal environment was included in the analyses models for birth weight, weaning weight and yearling weight, and the random effect of maternal genetics only in the model for weaning weight. The heritability estimates, in multivariate analysis for weights at birth, weaning, yearling and at 2,3 and 5 years of age were $0.25 ; 0.33 ; 0.34 ; 0.32 ; 0.33$ and 0.35 , respectively. The genetic correlations estimated between traits were positive and moderate to high in magnitude and tended to decrease with the increase in the distance between records, that indicated that selection for weight at any age will promote genetic change in weights at other ages including the female mature weight of the Nellore breed. The heritability estimates obtained in the multivariate analysis were high, especially at 3 and 5 years of age, suggesting better partition of genetic and environmental variances compared to uni and bivariate analyses but, high computer demands can still hinder its use in large databases.

Key Words: beef cattle, body weight, genetic correlation, weight at maturity 


\section{Introdução}

A tendência atual do mercado é buscar animais com maior velocidade de crescimento, os quais permanecem menos tempo em pastagens ou confinamentos, encurtando o ciclo de produção, o que possibilita maior retorno econômico. Portanto, a seleção de animais com maiores pesos em idades jovens é uma prática comum nos programas de melhoramento de bovinos de corte. Nos últimos anos, a seleção para maiores pesos ou ganhos de peso em idades jovens tem sido questionada com o argumento de que pode promover aumento do peso adulto dos animais, acarretando maiores custos de manutenção das matrizes, o que poderia reduzir as vantagens econômicas obtidas com o aumento de peso dos animais de abate (Bullock et al., 1993).

Os registros de peso adulto em um programa de melhoramento genético constituem amostra bastante selecionada e estão sujeitos aos efeitos de seleção, uma vez que são obtidos apenas em parte dos animais nascidos, geralmente nas fêmeas em reprodução. Nesse contexto, as análises unicaracterísticas pressupõem descarte aleatório dos animais, enquanto as multicaracterísticas indicam descarte não-aleatório, explorando as correlações genéticas e ambientais entre uma medida tomada antes da seleção dos animais e os pesos adultos medidos subsequentemente (Meyer, 1995; Kaps et al., 1999), constituindo uma alternativa para avaliação genética do peso adulto (Mercadante et al., 2004).

Visando estimar parâmetros genéticos para características de crescimento, Albuquerque \& Meyer (2001), Nobre et al. (2003) e Dias et al. (2005) relataram maior herdabilidade para peso ao nascer $(0,26$ a 0,33$)$, diminuição nas estimativas até próximo ao período da desmama $(0,11$ a $0,16)$ e, posteriormente, aumento dessas estimativas de acordo com a idade ( 0,21 e 0,25 para peso aos 570 dias de idade e 0,20 para peso aos 683 dias de idade). Os estudos disponíveis sobre peso adulto de Bos indicus sugerem a existência de variabilidade genética suficiente para obtenção de mudança genética em programas de melhoramento (Rosa et al., 2001; Talhari et al., 2003; Mercadante et al., 2004), tanto com objetivo de diminuir, como de aumentar ou mantê-lo constante. Entretanto, faltam pesquisas sobre peso adulto de raças zebuínas em condições brasileiras, desde abordagens de quais pesos devem ser considerados na avaliação genética até a magnitude das correlações com pesos e ganhos de peso obtidos em idades jovens.

Os objetivos neste estudo foram estimar herdabilidades e correlações genéticas para pesos do nascimento à idade adulta (peso ao nascer, à desmama, ao sobreano e aos 2, 3 e 5 anos de idade) para animais da raça Nelore, em modelos uni, bi e multicaracterísticos, com a finalidade de fornecer subsídios a programas de melhoramento genético da raça Nelore que fazem seleção para características de crescimento.

\section{Material e Métodos}

Utilizaram-se dados de 19.458 animais da raça Nelore, nascidos entre 1975 e 2002, pertencentes a oito fazendas participantes do Programa de Melhoramento Genético da Raça Nelore (PMGRN), que teve início em 1987. Os animais participantes, além de serem identificados de forma a garantir as informações de genealogia, são submetidos a pesagens a cada 90 dias, do nascimento ao sobreano, e aqueles que permanecem nos rebanhos como reprodutores continuam sendo pesados rotineiramente nesse intervalo. Neste conjunto de dados, os nascimentos ocorreram durante o ano todo, com maior concentração na primavera e no verão, e os animais foram desmamados, em média, aos 240 dias de idade. Do conjunto total de dados disponibilizados, foram mantidos apenas os pesos de animais: produtos de inseminação artificial, criados em pastagem sem suplementação, com peso ao nascer igual ou maior que $24 \mathrm{~kg}$, amamentados por suas mães biológicas e filhos de vacas que tinham entre 2 e 25 anos ao parto.

As características avaliadas foram os pesos ao nascimento, à desmama, ao sobreano e aos 2, 3 e 5 anos de idade. Para o peso à desmama, foi considerado o peso mais próximo aos 240 dias de idade, num intervalo de 180 a 300 dias, e, para o peso ao sobreano, o peso mais próximo aos 550 dias de idade, num intervalo de 490 a 610 dias. Para o peso aos 2 anos de idade, foi utilizado o peso mais próximo aos 2 anos de idade, num intervalo de 650 a 810 dias. Para os pesos aos 3 e aos 5 anos de idade, foi considerado o peso mais próximo dessas idades, nos intervalos dos 2,5 aos 3,5 anos e dos 4,5 aos 5,5 anos, respectivamente. Foram excluídos registros de pesos fora dos intervalos determinados pela média do grupo de contemporâneo mais ou menos três desvios-padrão. Como sugerido por Silva et al. (2000) e Rosa et al. (2001), o peso mais próximo aos 5 anos de idade foi considerado indicativo do peso adulto.

Foram consideradas quatro estações de nascimento, agrupando-se os meses de dezembro a fevereiro (estação 1), março a maio (estação 2), junho a agosto (estação 3) e setembro a novembro (estação 4). Os grupos de contemporâneos utilizados nas análises foram constituídos por animais nascidos na mesma fazenda, em mesmo ano e estação e pertencentes ao mesmo sexo. Para os pesos aos 3 e aos 5 anos de idade, não foi considerado o sexo no grupo de contemporâneos, uma vez que foram analisadas somente 
informações de fêmeas. Grupos de contemporâneos com menos de quatro observações foram excluídos (Tabela 1).

As variâncias e covariâncias genéticas aditivas e residuais foram estimadas pelo método da máxima verossimilhança restrita (REML) em análises uni, bi e multicaracterísticas. As análises bicaracterísticas foram realizadas sempre com o peso à desmama (PD) com objetivo de reduzir os efeitos da seleção sequencial. Para as análises uni e bicaracterísticas, foi utilizado o software MTDFREML (Boldman et al., 1995) e para a análise multicaracterística, o programa REMLF90 (Misztal, 2001), que emprega o algoritmo de Maximização da Esperança (EM), com processo de aceleração da convergência. Admitiu-se que a convergência era atingida quando o quadrado das diferenças relativas entre estimativas consecutivas era menor que $10^{-9}$.

Inicialmente, foram considerados pesos em nove idades diferentes (nascimento, desmama, ano, sobreano e aos 2, 3, 4, 5 e 6 anos de idade) em análises uni e bicaracterísticas. Entretanto, foi necessário retirar alguns pesos, uma vez que, após determinado número de interações, as matrizes tornavam-se negativas definidas.

Os modelos utilizados incluíram os efeitos genético aditivo direto, genético aditivo materno materno (para os pesos ao nascer, à desmama $e$ ao sobreano), de ambiente permanente materno e residual como aleatórios, além dos efeitos fixos de grupo de contemporâneos e os efeitos linear e quadrático da idade do animal à pesagem (com exceção do peso ao nascer) e da idade da mãe ao parto. Em todas as análises, foi utilizado um arquivo de genealogia contendo identificação do animal, pai e mãe, totalizando 26.924 animais na matriz de parentesco, considerando todas as relações de parentesco disponíveis (até 16 gerações).

\section{Resultados e Discussão}

O menor número de observações (Figura 1), obtidas principalmente a partir do sobreano, se deve ao fato de os animais terem sido selecionados da desmama ao sobreano e, a partir dessa idade, terem permanecido nos rebanhos somente aqueles usados como reprodutores. Para peso à desmama, peso ao sobreano, peso aos 2 anos de idade, os machos totalizavam 52, 45 e 34\% dos animais avaliados, respectivamente. A primeira monta das fêmeas ocorre, em média, aos 24 meses de idade e as que não emprenham na estação de monta ou desmamam bezerros são descartadas.

As médias de peso variaram de $32 \mathrm{~kg}$ ao nascimento até $514 \mathrm{~kg}$ aos 5 anos de idade (Figura 1). O peso apresentou aumento praticamente linear com a idade do animal até a desmama, mas após este período houve pequena redução na taxa de ganho de peso. A tendência de o peso continuar aumentando até os 5 anos de idade também foi observada por Mercadante et al. (2004) em fêmeas da raça Nelore. Estudando curva de crescimento de fêmeas da raça Nelore, Forni et al. (2007) observaram que o peso à maturidade foi atingido aos 4 anos.

As estimativas de variância genética aditiva nas análises bi e multivariadas foram superiores às estimadas em análises unicaracterísticas (Figura 2), principalmente para os pesos a partir do sobreano. Esse aumento na diferença do componente de variância genético aditivo nos modelos reflete a remoção do vício decorrente da seleção (Meyer, 1995) que ocorre com os modelos bi e multicaracterísticos. Nos rebanhos que geraram os dados analisados, a seleção foi praticada com base nos pesos da desmama ao sobreano, portanto, essas informações são essenciais para análise de caracteres obtidos após esta idade, como o peso adulto. Segundo Meyer (1995) e Kaps et al. (1999), em rebanhos em

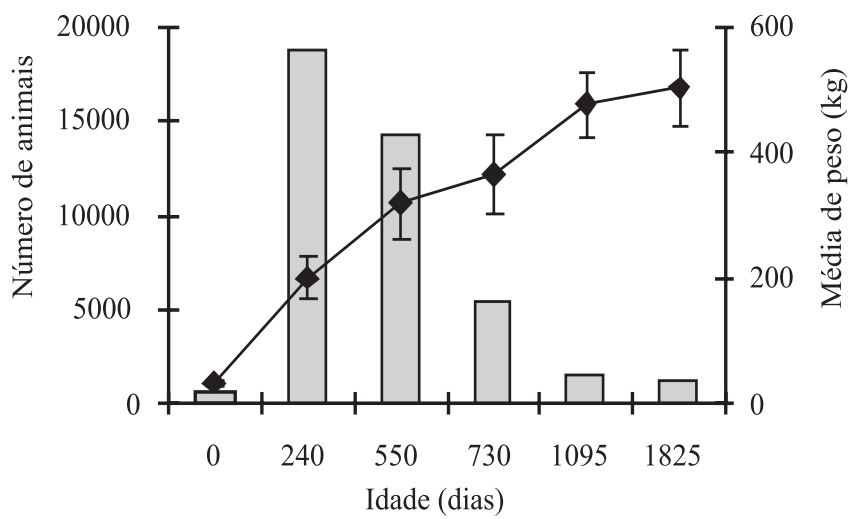

Figura 1 - Número de animais (barras), média e desvio-padrão de pesos (linhas) por idade.

Tabela 1 - Descrição dos dados dos pesos ao nascimento, à desmama, ao sobreano e aos 2, 3 e 5 anos de idade

\begin{tabular}{lcccccc}
\hline Descrição & $\begin{array}{c}\text { Peso ao } \\
\text { nascer }\end{array}$ & $\begin{array}{c}\text { Peso à } \\
\text { desmama }\end{array}$ & $\begin{array}{c}\text { Peso ao } \\
\text { sobreano }\end{array}$ & $\begin{array}{c}\text { Peso aos 2 anos } \\
\text { de idade }\end{array}$ & $\begin{array}{c}\text { Peso aos } 3 \text { anos } \\
\text { de idade }\end{array}$ & $\begin{array}{c}\text { Peso aos } 5 \text { anos } \\
\text { de idade }\end{array}$ \\
\hline Observações & 635 & 18.770 & 14.242 & 5.378 & 1.561 & 1.222 \\
Touros & 27 & 515 & 496 & 373 & 180 & 190 \\
Vacas & 397 & 8.546 & 7.382 & 3.756 & 1.115 & 966 \\
Grupo de contemporâneos & 55 & 490 & 411 & 249 & 114 & 115 \\
\hline
\end{tabular}



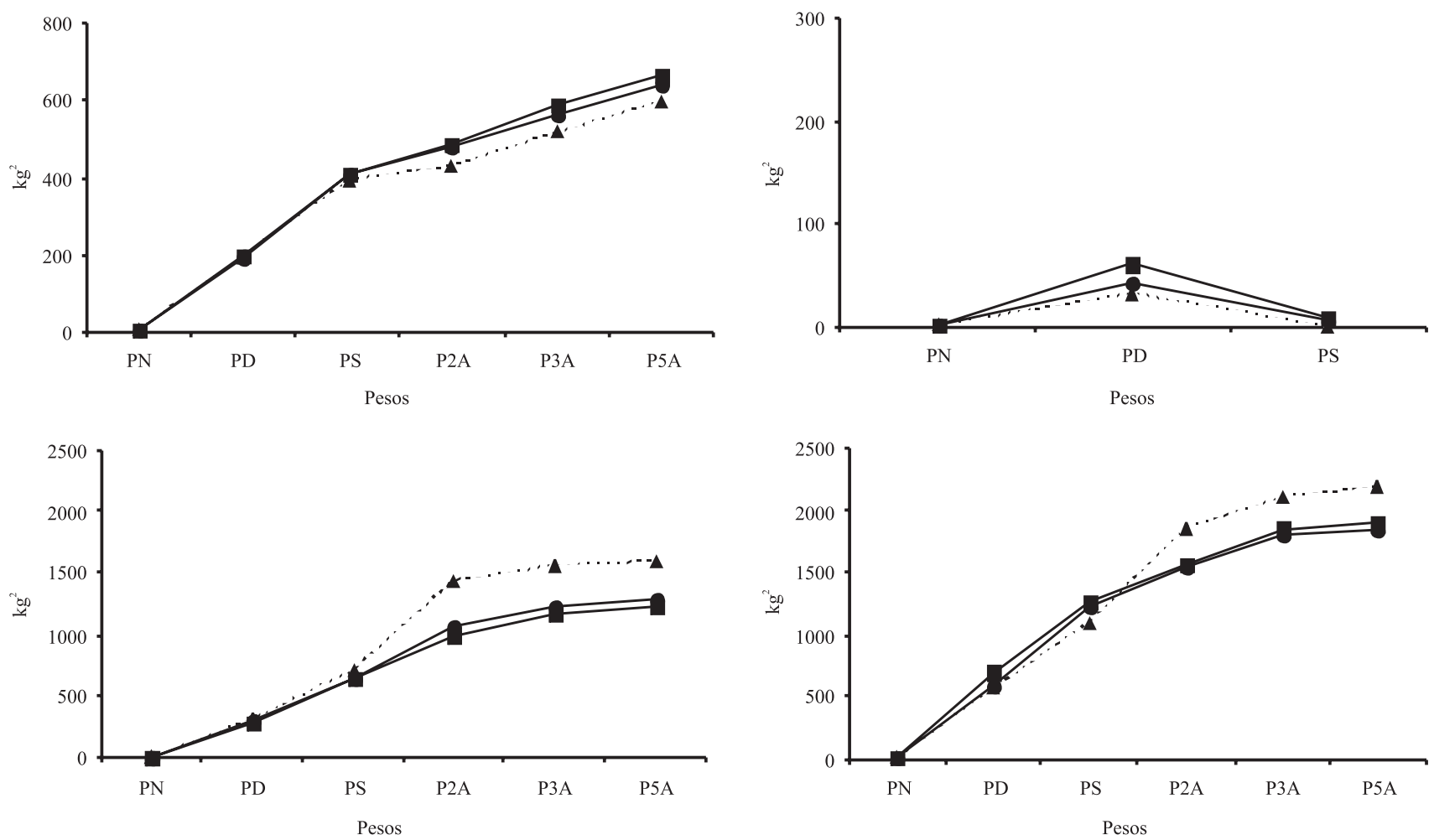

Figura 2 - Variâncias genética aditiva (acima à esquerda), de ambiente permanente materno (acima à direita), residual (abaixo à direita) e fenotípica (abaixo à esquerda) estimadas em análises uni $(\mathbf{\Lambda})$, bi $(\mathbf{O})$ e multicaracterísticas $(\boldsymbol{\square})$ para pesos ao nascer (PN), à desmama (PD), ao sobreano (PS) e aos 2 anos (P2A), aos 3 anos (P3A) e aos 5 anos (P5A) de idade.

que há, por razões econômicas, uma pré-seleção ao desmame, e a seleção propriamente dita ao ano ou sobreano, esses dados devem ser incluídos nas análises do peso adulto para considerar o efeito da seleção.

Os resultados deste trabalho confirmam os relatados por Mercadante et al. (2004), que observaram aumento na estimativa da variância genética aditiva do peso adulto quando avaliado em análise trivariada com os pesos à seleção (ao ano e ao sobreano). Do mesmo modo, avaliando pesos ao nascimento, à desmama, ao ano e à maturidade, Meyer et al. (1993) observaram aumento nas estimativas da variância genética aditiva em análises envolvendo três ou quatro pesos simultaneamente em relação às análises unicaracterísticas.

As contribuições das variâncias atribuídas ao ambiente permanente materno para a variância fenotípica foram mais expressivas em animais jovens e, em análise multicaracterística, foram de 18,6 e $0,5 \%$ para os pesos ao nascer, à desmama e ao sobreano, respectivamente (Figura 2), uma vez que os animais têm maior influência da mãe até a desmama e essa influência tende a desaparecer no período pós-desmama (Meyer et al., 1993; Mucari \& Oliveira, 2003; Dias et al., 2005). Semelhante ao obtido neste estudo, Albuquerque \& Meyer (2001) observaram em animais da raça Nelore que as estimativas de variância de ambiente permanente materno aumentaram até os 150 dias de idade e mantiveram-se praticamente constantes até os 240 dias, diminuindo após esta idade.

As estimativas da variância residual para os pesos pós-desmama foram mais altas nas análises unicaracterísticas em comparação às estimadas em modelos bi e multicaracterísticos (Figura 2). As estimativas de variância residual obtidas nas análises uni, bi e multivariadas foram semelhantes para os pesos do nascimento ao sobreano, pelo fato de os pesos à desmama e ao sobreano conterem a maior parte das informações que determinaram a seleção nesses rebanhos. A partir do sobreano, houve aumento nas diferenças entre as estimativas das variâncias residuais nos modelos bi e multicaracterísticos em relação ao unicaracterístico. Esse resultado indica a importância da inclusão de pesos utilizados como critérios de seleção em análises de pesos obtidos em idades posteriores, como é o caso do peso adulto.

Para os pesos ao nascer e à desmama, as diferenças nas variâncias fenotípicas nos modelos uni e bicaracterísticos 
foram pequenas (Figura 2), mas, a partir dos 2 anos de idade, foram maiores entre esses dois modelos, o que pode refletir o fato de a variância fenotípica, a partir desta idade, ser dependente somente das variâncias genética aditiva e residual, sem efeitos maternos. Mesmo com o aumento no valor da estimativa de variância genética aditiva nos modelos bi e multicaracterísticos, em virtude da recuperação de parte da variância perdida com a seleção, houve redução mais expressiva na variância residual, assim, a variância fenotípica estimada foi menor nesses modelos.

As herdabilidades estimadas para peso ao nascer e à desmama em análises uni, bi e multivariadas foram semelhantes (Figura 3) pelo fato de não haver seleção até o peso à desmama, o que sugere que os componentes de variância para esse peso podem ser obtidos em análise unicaracterística. Este resultado confirma o relatado por Meyer et al. (1993), os quais estimaram herdabilidade variando de 0,43 a 0,45 e de 0,19 a 0,21 para os pesos ao nascer e à desmama, respectivamente, utilizando análises uni, bi e multivariadas.

A herdabilidade estimada para o peso ao nascimento está abaixo da relatada por Albuquerque \& Meyer (2001), Sakaguti et al. (2003) e Dias et al. (2005), as quais variaram de 0,26 a 0,37. A baixa herdabilidade estimada neste estudo pode ser atribuída ao menor número de observações disponíveis nessa idade, o que dificultou a estimação da variabilidade genética aditiva presente na população para esta característica. A herdabilidade estimada para peso à desmama, no entanto, está de acordo com os valores descritos na literatura para bovinos de raças zebuínas, os quais variam de 0,23 a 0,45 (Ferraz Filho et al., 2002; Sakaguti et al., 2003; Talhari et al., 2003; Boligon et al., 2008), e sugerem que parte considerável da variação existente entre os animais para esta característica está sob influência de componente genético aditivo, dessa forma, o peso à desmama pode responder de forma satisfatória à seleção massal.

De modo semelhante, as estimativas de herdabilidade do efeito genético materno para peso à desmama foram semelhantes nas análises realizadas: de 0,16 (uni e bicaracterística) e 0,14 (multivariada), próximas à amplitude de 0,11 a 0,14 descrita na literatura (Albuquerque \& Meyer, 2001; Nobre et al., 2003; Dias et al., 2005; Boligon et al., 2008). Segundo Dias et al. (2005), a não-inclusão dos efeitos maternos nos modelos de análise para peso à desmama pode fazer com que a variância genética materna seja incluída na variância do efeito genético direto, resultando na superestimação desse efeito.

As herdabilidades estimadas para pesos após o peso ao sobreano foram maiores nas análises bi e multivariadas
(Figura 3). Como o peso à desmama é o que contém a maior parte das informações que determinam a seleção, já que não há seleção até essa idade, análises bicaracterísticas com o peso aos 240 dias de idade ou análise multivariada são mais apropriadas para avaliações de pesos pós-desmama, pois removem o viés causado pelo efeito da seleção. Meyer(1995) e Kaps et al. (1999) também observaram aumento significativo nas herdabilidades para peso final e adulto, respectivamente, quando medidas tomadas antes da seleção eram incluídas nas análises multicaracterísticas. Portanto, com análises multicaracterísticas, estimam-se as variâncias genética aditiva e residual para cada idade levando em conta as correlações entre todas as idades, ou seja, do nascimento aos 5 anos. Isso pode explicar o fato de a herdabilidade estimada para peso ao sobreano ter sido maior na análise unicaracterística em relação às demais, uma vez que as variâncias estariam mais sujeitas aos efeitos de amostragem.

As herdabilidades estimadas para peso aos 2, 3 e 5 anos de idade em análise multivariada foram de 0,32; 0,33 e 0,35 , respectivamente. Ressalta-se que estimativas de parâmetros para pesos aos 2, 3 e 5 anos de idade, principalmente em Bos indicus, são escassas na literatura. Rosa et al. (2001) relataram herdabilidade de 0,26 para peso adulto de fêmeas Nelore, o qual foi definido como o primeiro peso a partir dos 4 anos de idade. Considerando o último peso na entrada da estação de monta como indicador de peso adulto de matrizes Nelore, Mercadante et al. (2004) estimaram herdabilidade de 0,34 (incluindo vacas de 2 e 3 anos de idade) e 0,42 (considerando somente vacas a partir de 4 anos de idade) em análises multicaracterísticas.

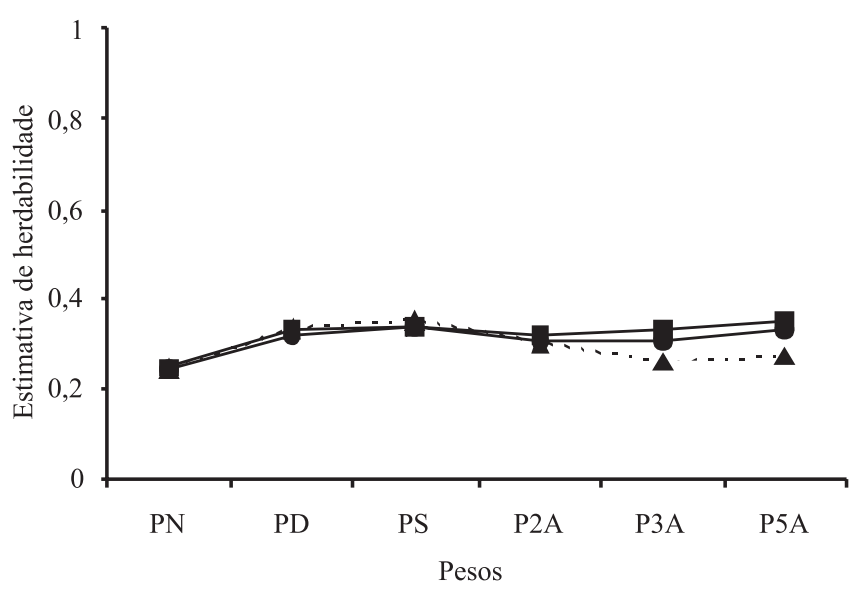

Figura 3 - Estimativas de herdabilidade para peso ao nascer $(\mathrm{PN})$, à desmama (PD), ao sobreano (PS) e aos 2, 3 e 5 anos de idade, obtidas em análises uni $(\mathbf{\Lambda})$, bi $(\mathbf{O})$ e multicaracterísticas ( $\square$ ). 
As correlações genéticas estimadas entre o peso ao nascer e pesos até os dois anos de idade foram positivas, de moderadas a altas magnitudes, com tendência de diminuição após esse período (Tabela 2). Portanto, a seleção baseada em pesos obtidos em idades jovens pode levar ao aumento do peso ao nascimento e provocar aumento da incidência de partos distócicos.

As correlações genéticas estimadas entre peso à desmama, peso ao sobreano e peso aos 2 anos de idade foram altas e positivas e indicam que os genes responsáveis por maiores pesos da desmama aos 2 anos de idade são, na maior parte, os mesmos. Esses resultados estão de acordo com os descritos por Ferraz Filho et al. (2002), Malhado et al. (2002), Santos et al. (2005) e Boligon et al. (2008), que variaram de 0,61 a 0,83 entre peso à desmama e peso ao sobreano. Em análise multicaracterística, Meyer et al. (1993) estimaram correlação genética de 0,92 entre peso à desmama e peso ajustado aos 600 dias de idade. Por outro lado, trabalhando com pesos às mesmas idades que as deste estudo, Mucari \& Oliveira (2003) relataram correlações genéticas inferiores.

As correlações genéticas estimadas entre pesos obtidos em idades jovens com peso adulto variaram de moderadas a altas. Para animais da raça Hereford, Meyer et al. (2004) estimaram correlações genéticas entre pesos ao nascimento, à demama e ao sobreano e peso adulto de 0,62; 0,49 e 0,77, respectivamente. De modo semelhante, Bullock et al. (1993) obtiveram estimativas de correlações genéticas de 0,64;
0,80 e 0,89 entre peso adulto e peso ao nascimento, à desmama e ao ano, respectivamente. Em geral, as correlações genéticas são maiores entre pesos adjacentes e reduzem à medida que as idades se distanciam. Os valores de correlações genéticas sugerem que animais geneticamente superiores em determinada idade deverão em grande parte ser superiores também nas idades posteriores, principalmente a partir da desmama.

$\mathrm{Na}$ comparação empírica de modelos uni, bi e multicaracterísticos utilizados para avaliar pesos do nascimento à idade adulta, o modelo multicaracterístico parece ser o mais indicado quando o objetivo for avaliação genética do peso adulto. Entretanto, uma das limitações na utilização de modelo multicaracterístico é que nem sempre podem ser analisados pesos em todas as idades disponíveis.

De modo geral, as correlações genéticas estimadas para pesos em idades jovens e os pesos em idades mais avançadas podem ser consideradas de relevante valor prático, uma vez que o peso adulto estaria sujeito a aumentos acentuados, como resposta à seleção para maiores pesos em idades mais jovens, o que vem sendo praticado em gado de corte. Esses resultados reforçam a preocupação atual com o tamanho adulto de matrizes para a maioria das raças bovinas de corte, uma vez que, segundo alguns estudos, existe correlação genética baixa, porém desfavorável, entre peso adulto e características reprodutivas (Meyer et al., 2004; Mello et al., 2006).

Tabela 2 - Estimativas das correlações genéticas (acima da diagonal) e residual (abaixo da diagonal) entre as características de crescimento obtidas em análise multicaracterística

\begin{tabular}{|c|c|c|c|c|c|c|}
\hline & $\begin{array}{l}\text { Peso ao } \\
\text { nascer }\end{array}$ & $\begin{array}{c}\text { Peso à } \\
\text { desmama }\end{array}$ & $\begin{array}{l}\text { Peso ao } \\
\text { sobreano }\end{array}$ & $\begin{array}{c}\text { Peso aos } 2 \text { anos } \\
\text { de idade }\end{array}$ & $\begin{array}{c}\text { Peso aos } 3 \text { anos } \\
\text { de idade }\end{array}$ & $\begin{array}{c}\text { Peso aos } 5 \text { anos } \\
\text { de idade }\end{array}$ \\
\hline Peso ao nascer & - & 0,81 & 0,74 & 0,61 & 0,54 & 0,43 \\
\hline Peso à desmama & 0,27 & - & 0,82 & 0,69 & 0,65 & 0,63 \\
\hline Peso ao sobreano & 0,26 & 0,41 & - & 0,85 & 0,69 & 0,66 \\
\hline Peso aos 2 anos de idade & 0,19 & 0,32 & 0,37 & - & 0,71 & 0,74 \\
\hline Peso aos 3 anos de idade & 0,12 & 0,24 & 0,28 & 0,31 & - & 0,88 \\
\hline Peso aos 5 anos de idade & 0,10 & 0,19 & 0,17 & 0,26 & 0,39 & - \\
\hline
\end{tabular}

\section{Conclusões}

A seleção com base em características de crescimento em qualquer idade pode promover ganhos genéticos significativos no peso corporal de animais da raça Nelore, em todas as idades-padrão, inclusive nos pesos ao nascer e à idade adulta das fêmeas. O modelo multicaracterístico, incluindo registros de pesos ao desmame e à seleção, é o mais indicado para a avaliação genética de pesos pósdesmame.

\section{Literatura Citada}

ALBUQUERQUE, L.G.; MEYER, K. Estimates of direct and maternal genetic effects for weights from birth to 600 days of age in Nelore cattle. Journal of Animal Breeding and Genetics, v.118, p.83-92, 2001.

BOLDMAN, K.G.; KRIESE, L.A.; VAN VLECK, L.D. et al. A manual for use of MTDFREML: a set of programs to obtain estimates of variance and (co)variance (DRAFT). Lincoln: Department of Agriculture/ARS, 1995. 120p.

BOLIGON, A.A.; ALBUQUERQUE, L.G.; RORATO, P.R.N. Correlações genéticas entre pesos e características reprodutivas 
de fêmeas da raça Nelore. Revista Brasileira de Zootecnia, v.37, n.4, p.596-601, 2008.

BULLOCK, K.D.; BERTRAND, J.K.; BENYSHERK, L.L. Genetic and environmental parameters for mature weight and other growth measures in Polled Hereford cattle. Journal of Animal Science, v.71, n.7, p.1737-1741, 1993.

DIAS, L.T.; ALBUQUERQUE, L.G.; TONHATI, H. et al. Estimação de parâmetros genéticos para peso em diferentes idades para animais da raça Tabapuã. Revista Brasileira de Zootecnia, v.34, n.6, p.1914-1919, 2005.

FERRAZ FILHO, P.B.; RAMOS, A.A.; SILVA, L.O.C. et al. Herdabilidade e correlações genéticas, fenotípicas e ambientais para pesos em diferentes idades de bovinos da raça Tabapuã. Archives of Veterinary Science, v.7, n.1, p.65-69, 2002.

FORNI, S.; PILES, M.; BLASCO, A. et al. Analysis of beef cattle longitudinal data applying a non-linear model. Journal of Animal Science, v.85, p.3189-3197, 2007.

KAPS, M.; HERRING, W.O.; LAMBERSON, W.R. Genetic and environmental parameters for mature weight in Angus cattle. Journal of Animal Science, v.77, p.569-574, 1999.

MALHADO, C.H.M.; SOUZA, J.C.; SILVA, L.O.C. et al. Correlações genéticas, fenotípicas e de ambiente entre os pesos de várias idades em bovinos da raça Guzerá no estado de São Paulo. Archives of Veterinary Science, v.7, n.1, p.71-75, 2002.

MELLO, S.P.; ALENCAR, M.M.; TORAL, F.L.B. et al. Estimativas de parâmetros genéticos para características de crescimento e produtividade em vacas da raça Canchim, utilizando-se inferência bayesiana. Revista Brasileira de Zootecnia, v.35, n.1, p.92-97, 2006.

MERCADANTE, M.E.Z.; RAZOOK, A.G.; TROVO, J.B.F. et al. Parâmetros genéticos do peso no início da estação de monta, considerando indicativo do peso adulto de matrizes Nelore. Revista Brasileira de Zootecnia, v.33, n.5, p.1135-1144, 2004.

MEYER, K.; CARRICK, M.J.; DONNELLY, B.J.P. Genetic parameters for growth traits of Australian beef cattle from a multibreed selection experiment. Journal of Animal Science, v.71, p.2614-2622, 1993.
MEYER, K. Estimates of genetic parameters for mature weight of Australian beef cows and its relationship to early growth and skeletal measures. Livestock Production Science, v.44, p.125-137, 1995.

MEYER, K.; JOHNSTON, D.; GRASER, H. Estimates of the complete genetic covariance matrix for traits in multi-trait genetic evaluation of Australian Hereford cattle. Australian Journal of Agricultural Research, v.55, p.195-210, 2004.

MISZTAL, I. [2001]. REMLF90 Manual. Disponível em: <http:// nce.ads.uga.edu/ ignacy/newprograms.html/>. Acesso em 6/4/2007.

MUCARI, T.B.; OLIVEIRA, J.A. Análise genético-quantitativa de pesos aos 8, 12, 18 e 24 meses de idade em um rebanho da raça Guzerá. Revista Brasileira de Zootecnia, v.32, n.6, p.1604-1613, 2003.

NOBRE, P.R.C.; MISZTAL, I.; TSURUTA, S. et al. Analyses of growth curves of Nellore cattle by multiple-trait and random regression models. Journal of Animal Science, v.81, p.918-926, 2003.

ROSA, A.N.; LÔBO, R.B.; OLIVEIRA, H.N. et al. Peso adulto de matrizes em rebanhos de seleção da raça Nelore no Brasil. Revista Brasileira de Zootecnia, v.30, n.3, p.1027-1036, 2001.

SAKAGUTI, E.S.; SILVA, M.A.; QUAAS, R.L. et al. Avaliação do crescimento de bovinos jovens da raça Tabapuã, por meio de análises de funções de covariância. Revista Brasileira de Zootecnia, v.32, n.4, p.864-874, 2003.

SANTOS, P.F.; MALHADO, C.H.M.M.; CARNEIRO, P.L.S. et al. Correlação genética, fenotípica e ambiental em características de crescimento de bovinos da raça Nelore variedade mocha. Archives of Veterinary Science, v.10, n.2, p.55-60, 2005.

SILVA, A.M.; ALENCAR, M.M.; FREITAS, A.R. et al. Herdabilidades e correlações genéticas para peso e perímetro escrotal de machos e características reprodutivas e de crescimento de fêmeas, na raça Canchim. Revista Brasileira de Zootecnia, v.29, n.6, p.2223-2230, 2000.

TALHARI, F.M.; ALENCAR, M.M.; MASCIOLI, A.S. et al. Correlações genéticas entre características produtivas de fêmeas em um rebanho da raça Canchim. Revista Brasileira de Zootecnia, v.32, n.4, p.880-886, 2003. 